\title{
Muscular atrophy in diabetic neuropathy: a stereological magnetic resonance imaging study
}

\author{
H . A ndersen ${ }^{1}$, P. C. G adeberg ${ }^{1}$, B . B rock ${ }^{2}$, J . J akobsen ${ }^{1}$ \\ ${ }^{1}$ Department of Neurology, Aarhus University Hospital, Aarhus, Denmark \\ ${ }^{2}$ Department of Endocrinology and Metabolism, Aarhus University Hospital, Aarhus, Denmark
}

Summary Diabetic patients with polyneuropathy develop motor dysfunction. To establish whether motor dysfunction is associated with muscular atrophy the ankle dorsal and plantar flexors of the non-dominant leg were evaluated with magnetic resonance imaging in 8 patients with symptomatic neuropathy, in 8 nonneuropathic patients and in 16 individually matched control subjects. In the neuropathic patients the muscle strength of the ankle dorsal and plantar flexors was reduced by $41 \%$ as compared to the non-neuropathic patients $(p<0.005)$. Volume of the ankle dorsal and plantar flexors was estimated with stereological techniques from consecutive cross-sectional images of the lower leg. The neuropathic patients had a $32 \%$ reduction in volume as compared with the nonneuropathic patients $(p<0.005)$. To determine the regional distribution of atrophy cross-sectional magnetic resonance images were performed at predetermined levels of the lower leg in relation to bone landmarks. In the neuropathic patients there was an insignificant increase of $3 \%$ of muscle area at the proximal lower leg level, whereas the atrophy was $43 \%(p<0.002)$ at the mid lower leg level and $65 \%(p<0.002)$ distally. Analysis of individual muscles confirmed that the atrophy predominated distally. We conclude that muscular atrophy underlies motor weakness at the ankle in diabetic patients with polyneuropathy and that the atrophy is most pronounced in distal muscles of the lower leg indicating that a length dependent neuropathic process explains the motor dysfunction. [Diabetologia (1997) 40: 1062-1069]

Keywords Muscular atrophy, diabetic neuropathy, MR-imaging, stereology, motor dysfunction.
Motor dysfunction develops in advanced cases of diabetic polyneuropathy $[1,2]$. In the clinic it is most frequently detected by inability to walk on heels which indicates a preponderance for the dorsal flexors of the ankle. Recently weakness of both the ankle dorsal and plantar flexors and of the knee extensors and

Received: 17 January 1997 and in revised form: 24 April 1997

Corresponding author: H. Andersen M.D., Department of Neurology, Aarhus University Hospital, Nørrebrogade 44, DK-8000 Aarhus C, Denmark

A bbreviations: IDDM, Insulin-dependent diabetes mellitus; MRI, magnetic resonance imaging; NDS, neurological disability score; NSS, neuropathy symptom score; MNCV, motor nerve conduction velocity; SNCV, sensory nerve conduction velocity; CSA, cross-sectional area. flexors was found in long-term insulin-dependent diabetic (IDDM) patients with peripheral neuropathy at isokinetic dynamometry [3]. Furthermore, the weakness at the ankle and knee was closely related to the severity of neuropathy assessed clinically, electrophysiologically and at quantitative sensory examination.

The present study was performed in order to clarify whether muscle weakness in diabetic patients with polyneuropathy is associated with muscular atrophy and secondly if present how the atrophy is topographically distributed. A non-invasive quantitative method for analysis of muscle volume was applied in the present study. Assessment of muscle morphology can be obtained with ultrasonomyography, X-ray computed tomography (CT), and magnetic resonance imaging (MRI) [4-7]. Ultrasonomyography is 
fast and inexpensive but has a low spatial resolution [4] and CT has a low tissue contrast and involves radiation exposure [4]. MRI has the highest resolution and can be combined with stereological techniques enabling a quick and reliable estimation of volume of any compartment of the body [8]. The present study applied MRI to evaluate muscle structure and volume in neuropathic and non-neuropathic longterm IDDM patients in whom maximal isokinetic muscle strength was determined.

\section{Subjects, materials and methods}

Patients and control subjects. Eight IDDM patients with symptomatic neuropathy and weakness of the thigh and lower leg muscles, 8 IDDM patients without neuropathy and 16 healthy age-, sex-, height-, and weight-matched control subjects were included in the study. Patients were not included if they had severe cardiac or lung disease, acute or chronic musculo-skeletal disease, any other neurological or endocrine disease, symptomatic macroangiopathy or any previous or present lower limb asymmetric proximal weakness. None had had foot ulcers, in all patients the pulse of the dorsal foot artery was palpable and all neuropathic patients had findings typical of diabetic polyneuropathy. Patients were classified according to their urinary albumin excretion: less than $20 \mu \mathrm{g} / \mathrm{min}$ (normoalbuminuria), between 20 and $200 \mu \mathrm{g} / \mathrm{min}$ (incipient nephropathy), and over $200 \mu \mathrm{g} / \mathrm{min}$ (overt nephropathy). Furthermore the retinal status of the patients was classified as no, simplex, or proliferative retinopathy by an ophthalmologist. All subjects gave informed consent to the study which was approved by the local ethics committee.

Clinical evaluation, quantitative sensory examination, and electrophysiological studies. Patients were evaluated according to a neuropathy symptom score (NSS) [9] and a neurological disability score (NDS) by a trained neurologist [10]. The NSS is the number of symptoms of motor, sensory, and autonomic disturbances. The NDS is a combined score obtained from the neurological examination of muscle weakness, activity of tendon reflexes, and assessment of sensation on great toe and index finger. Vibratory perception threshold (VPT) was evaluated at dominant index finger pulp and non-dominant dorsum of the great toe using forced choice techniques (CASE IV; WR Medical Electronics Co., Stillwater, Minn., USA). The threshold was determined with the 4, 2, and 1 stepping algorithm [11]. The vibratory perception thresholds for each patient were compared with values of a large group of healthy subjects and the corresponding percentiles were determined. Nerve conduction studies were performed with an electromyograph as described elsewhere (Dantec Counterpoint, Skovlunde, Denmark) [12]. Motor nerve conduction velocity (MNCV) was measured in the dominant forearm segment of the median nerve and in the non-dominant leg segment of the peroneal nerve. Sensory nerve conduction velocity (SNCV) was measured in the non-dominant sural nerve with orthodromic activation and with antidromic activation of the dominant median nerve.

The external circumference of the non-dominant leg was measured at three levels of the lower leg and at two levels of the thigh. To ensure comparable measures the levels were defined in relation to bone landmarks. The lower leg was defined as the distance from the distal end of the lateral malleolus to the lateral articular cleft between the femur and tibia chondyles. Measurements were performed at levels 20, 50, and $80 \%$ of the distance from the lateral malleolus and designated as distal, mid, and proximal levels of the lower leg, respectively. The thigh length was defined as the distance from the lateral articular cleft between the femur and tibia chondyles to the upper end of the trochanter major. Measurement of the circumference was performed at levels 20 and $50 \%$ of the distance from the knee joint and designated as distal and mid levels of the thigh, respectively.

$M$ agnetic resonance imaging. The non-dominant leg of all subjects was evaluated with MRI at 1.5 Tesla (Phillips Gyroscan, Eindhoven, The Netherlands). The MR-scans were performed with the subject in a supine position on a couch placed inside the scanner. Subjects were moved between each MR-scan. All MR-scans were obtained with a conventional T1 weighted Spin-Echo sequence (Echo time $=20 \mathrm{~ms}$, Repetition time $=550 \mathrm{~ms}$ ) providing $5 \mathrm{~mm}$ cross-sectional MR-images. A $256 \times 256$ matrix and two excitations were used. The MR-images were converted to digital pictures for analysis on a PC (Chameleon; Olympus, Ballerup, Denmark). Muscular anatomy was defined in accordance with a standard anatomy textbook [13]. The tibialis anterior, extensor digitorum longus, extensor hallucis longus, and peroneus tertius muscles were defined as the ankle dorsal flexors. The triceps surae, plantaris, flexor hallucis longus, flexor digitorum longus, tibialis posterior, peroneus longus, and peroneus brevis muscles were defined as the ankle plantar flexors. The knee extensor was the quadriceps femoris muscle whereas the biceps femoris, semitendinosus, semimembranosus, sartorius and gracilis muscles were the knee flexors.

The identity of MR-images was blinded to the observers. Within the muscle compartments an upper level of signal intensity for muscle tissues was defined. Signal intensities above this level were comparable to signal intensities of fat tissue allowing a separation of muscle tissues from "non-muscle tissues" within the muscle compartments. Muscle fascia, tendons or blood vessels within muscle groups were excluded. Crosssectional areas (CSA) were estimated by a single observer with a stereological point counting method $[14,15]$. This is performed using a grid with a systematic array of test points (CAST-grid; Olympus) placed at random on the MR-images. The CSA is calculated by multiplying the number of muscle tissue points by the unit test point area.

M usclevolumeestimation. Volume estimation was based on stereological principles [15]. The object of interest is intersected by a series of parallel planes at a known distance apart (T). In all sections the CSAs of the muscle groups was estimated as described above. Volume is calculated by multiplying $\mathrm{T}$ by the total CSA, the first section being randomly placed within the interval length. For muscle volume estimation 14 systematic $5 \mathrm{~mm}$ thick scans were performed at fixed intervals using a random start section within the distance T. Since the field of view was smaller than the segment of interest, the subjects were moved once during each session by a computer controlled movement of the couch. Each examination consisted of two scanning sessions of 8 sections excluding overlapping sections. Unit test point areas in the range of $23-87 \mathrm{~mm}^{2}$ were used to ensure that 100-500 points were counted for each muscle group.

Cross-sectional muscle areas at predetermined levels. To determine the regional distribution of muscle tissues an MR-scan was performed at the five levels of the leg defined above where external circumference was measured. At each level the CSA of the muscle groups of interest was estimated. To detect 
Table 1. Clinical data for IDDM patients and control subjects

\begin{tabular}{|c|c|c|c|c|c|c|}
\hline & $\mathrm{n}$ & $\begin{array}{l}\text { Age } \\
\text { (years) }\end{array}$ & $\begin{array}{l}\text { Weight } \\
(\mathrm{kg})\end{array}$ & $\begin{array}{l}\text { Height } \\
(\mathrm{cm})\end{array}$ & $\begin{array}{l}\text { Retinopathy } \\
\text { (none, simplex, } \\
\text { proliferative) }\end{array}$ & $\begin{array}{l}\text { Nephropathy } \\
\text { (none, incipient, } \\
\text { overt) }\end{array}$ \\
\hline Neuropathic patients & 8 & $46.5(31-64)$ & $72(62-97)$ & $179(172-188)$ & $(0,2,6)$ & $(2,4,2)$ \\
\hline Non-neuropathic patients & 8 & $42.5(35-56)$ & $68(54-83)$ & $174(165-188)$ & $(4,4,0)$ & $(8,0,0)$ \\
\hline Control subjects & 8 & $40.5(34-56)$ & $67(53-78)$ & $170(162-184)$ & & \\
\hline
\end{tabular}

Values are median (range)

Table 2. Data of clinical findings (neurological disability score (NDS) and the neuropathy symptom score (NSS)), quantitative sensory examination (VPT), and motor nerve conduction velocity (MNCV) in the diabetic patients

\begin{tabular}{|c|c|c|c|c|c|c|c|}
\hline & $\begin{array}{l}\text { Duration of } \\
\text { diabetes } \\
\text { (years) }\end{array}$ & NDS & NSS & $\begin{array}{l}\text { VPT } \\
\text { hallux } \\
\text { (percentile) }\end{array}$ & $\begin{array}{l}\text { VPT } \\
\text { index finger } \\
\text { (percentile) }\end{array}$ & $\begin{array}{l}\text { MNCV } \\
\text { median } \\
\text { nerve }(\mathrm{m} / \mathrm{s})\end{array}$ & $\begin{array}{l}\text { MNCV } \\
\text { peroneal } \\
\text { nerve }(\mathrm{m} / \mathrm{s})\end{array}$ \\
\hline$\overline{\mathrm{Neu}}$ & $30(2$ & $34(27-46)$ & $3(1-7)$ & $>98$ & $>98$ & $46.0(41.0-52.1)$ & 27.1 \\
\hline
\end{tabular}

Values are median (range)

whether there was a difference in individual muscle morphology in proximal and distal parts of the lower leg the CSAs of the mid-portions of the medial gastrocnemius and flexor hallucis muscles were estimated. Unit test point areas of the same size as for volume estimation were used.

Isokinetic muscle testing. The maximal isokinetic muscle strength of ankle dorsal and plantar flexion, and knee extension and flexion of the non-dominant leg was evaluated with an isokinetic dynamometer (Lido Active Multijoint II; Loredan Biomedical Inc., Wast Sacramento, Calif., USA). All isokinetic testing procedures have been described in detail elsewhere $[3,16]$.

D efinitions and statistical analysis. The minimal criteria for diabetic neuropathy were adopted $[1,17]$. Patients were defined as neuropathic if at least 2 out of the following 4 categories were abnormal, the 1 being an abnormality either of nerve conduction or of sensory examination: (a) NSS 1 or more; (b) NDS 2 or more; (c) abnormal nerve conduction velocity in at least 2 out of 4 nerves; (d) abnormal VPT at index finger and great toe ( $\geq 98$ th percentile). In order to compare strength, muscle volume and muscle area of the neuropathic and the non-neuropathic patients, the value of each individual patient was expressed as a percentage of an individually matched control subject.

The primary study parameters were total muscle volume and total cross-sectional muscle tissue area at the distal, mid, and proximal levels of the lower leg. Differences between the neuropathic and non-neuropathic patients were statistically compared with an unpaired Student's t-test with a $5 \%$ Bonferroni corrected limit of significance. All other differences of the study were compared with an unpaired Student's t-test using a $5 \%$ limit of significance.

\section{Results}

Of the 8 neuropathic patients 3 had symptomatic weakness of the legs. All had loss of cutaneous sensation in the feet and 4 patients complained of leg pain. Detailed information about the basic clinical data of
Table 3. Maximal isokinetic muscle strength for the neuropathic and the non-neuropathic patients given as a percentage of control subjects

\begin{tabular}{lllll}
\hline & Ankle & & \multicolumn{2}{l}{ Knee } \\
\cline { 2 - 3 } \cline { 5 - 5 } & $\begin{array}{l}\text { Dorsal } \\
\text { flexion }\end{array}$ & $\begin{array}{c}\text { Plantar } \\
\text { flexion }\end{array}$ & Extension & Flexion \\
\hline $\begin{array}{l}\text { Neuropathic } \\
\text { patients }\end{array}$ & $52 \pm 13.6^{\mathrm{a}}$ & $55 \pm 13.5^{\mathrm{a}}$ & $73 \pm 19.0^{\mathrm{b}}$ & $72 \pm 18.2$ \\
$\begin{array}{l}\text { Non-neuropathic } \\
\text { patients }\end{array}$ & $87 \pm 23.6$ & $96 \pm 21.9$ & $98 \pm 18.9$ & $102 \pm 39.8$ \\
\hline
\end{tabular}

Values are mean \pm SD. Neuropathic patients are compared with non-neuropathic patients

${ }^{\mathrm{a}} \mathrm{p}<0.005 ;{ }^{\mathrm{b}} \mathrm{p}<0.05$

the patients and their matched control subjects are given in Table 1 . The MNCV of the peroneal nerve in neuropathic patients could be determined in 1 subject only, due to atrophy of the extensor digitorum brevis muscles. Information about the results of the electrophysiological, clinical, and sensory examination is given in Table 2. The neuropathic patients had a significant reduction in maximal muscle strength of ankle dorsal and plantar flexors as well as of knee extensors as compared to the non-neuropathic patients (Table 3). Leg circumference at the distal level of the lower leg was $92 \pm 6.5 \%$ and $99 \pm 8.3 \%$ (mean \pm SD) of the control subjects in the neuropathic and nonneuropathic group, respectively (ns). At the mid level of the lower leg, the leg circumference was significantly reduced in the neuropathic group compared to the non-neuropathic group being $89 \pm 6.6 \%$ and $101 \pm 10.7 \%$, respectively $(p<0.02)$. Neither at the proximal level of the lower leg nor at the thigh was there any difference of circumference.

The dorsal and the plantar flexor groups of the ankle as well as the knee extensor and flexor groups 


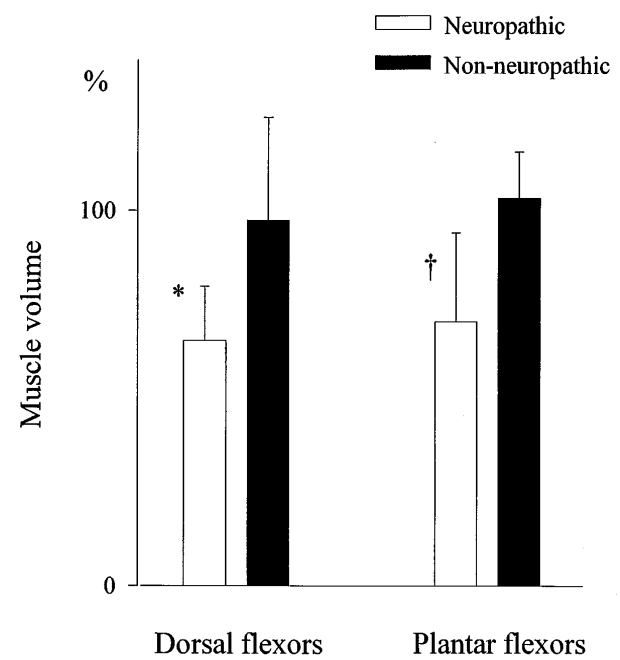

Fig. 1. Muscle volume (\% of matched control subjects) of the ankle dorsal and plantar flexors in neuropathic $\square$ and nonneuropathic $\square$ patients. Data are mean \pm SD. Values for neuropathic patients are compared with non-neuropathic patients. $* p<0.02 ; \dagger p<0.01$

could be identified at all cross-sectional MR-images. Volume of muscular tissues for the ankle dorsal and plantar flexors was significantly smaller for the neuropathic patients as compared to the non-neuropathic patients $(p<0.02)$ (Fig. 1). A progressive loss of muscular tissues in the proximo-distal direction was observed of the dorsal as well as of the plantar flexors (Fig.2). No such systematic differences were found for the non-neuropathic patients.

Studies of regional distribution of atrophy at predetermined levels showed areas of increased signal intensity within the muscle compartments at the middle and distal level of the lower leg in neuropathic patients (Fig. 3). The CSA of the dorsal and plantar flexors was significantly smaller at the distal and mid part of the lower leg in the neuropathic patients as compared to the non-neuropathic patients (Figs. 4 and 5). Following exclusion of high signal intensity areas at the same two levels the CSA of muscle tissues was further reduced (Figs. 4 and 5). In the thigh, no increased signal intensity was found within muscle compartments of the neuropathic patients. A slight reduction in CSA of the knee extensors and flexors
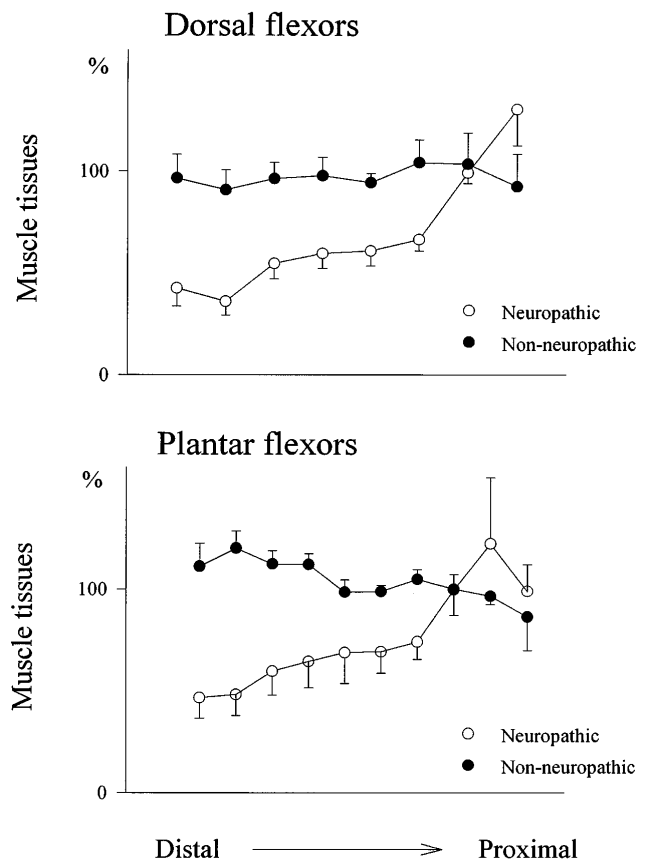

Fig. 2. Cross-sectional muscle area (\% of matched control subjects) of the dorsal flexors (above) and plantar flexors (below) from consecutive MR images in the neuropathic $\bigcirc$ and nonneuropathic patients. Data are mean \pm SEM

was found in the neuropathic group. This difference, however, did not reach statistical significance (Table 4).

Analysis of atrophy of individual muscles showed that at the proximal level the CSA of the medial gastrocnemius muscle was $87 \pm 18.2 \%$ (mean \pm SD) in the neuropathic group and $100 \pm 13.0 \%$ in the nonneuropathic group as compared to controls (NS). At the distal level, the CSA of the flexor hallucis longus muscle was $47 \pm 29.3 \%$ in the neuropathic group while it was $101 \pm 19.6 \%$ in the non-neuropathic group $(p<0.001)$.

The maximal muscle strength of all diabetic patients was related to the muscle volume for dorsal $(r=0.81, p<0.0002)$ and plantar flexors $(r=0.82$, $p<0.0001)$. The ratio of the strength to the corresponding muscle volume (the intrinsic strength) was reduced for the dorsal flexors in all diabetic patients $(p<0.02)$ as compared to the control subjects being
Table 4. Cross-sectional area of total muscle compartments (MC) and of muscle tissues after exclusion of high intensity values (MT) of knee extensors and flexors in the diabetic pa- tients at 2 levels of the thigh (distal and mid) given as a percentage of control subjects

\begin{tabular}{|c|c|c|c|c|c|c|c|c|}
\hline & \multicolumn{4}{|c|}{ Thigh-distal } & \multicolumn{4}{|l|}{ Thigh-mid } \\
\hline & \multicolumn{2}{|l|}{ Extensors } & \multicolumn{2}{|l|}{ Flexors } & \multicolumn{2}{|l|}{ Extensors } & \multicolumn{2}{|l|}{ Flexors } \\
\hline & $\mathrm{MC}$ & MT & $\mathrm{MC}$ & MT & $\mathrm{MC}$ & MT & $\mathrm{MC}$ & MT \\
\hline Neuropathic patients (8) & $87 \pm 6.2$ & $86 \pm 9.3$ & $84 \pm 16.0$ & $87 \pm 19.5$ & $85 \pm 7.4^{\mathrm{a}}$ & $85 \pm 8.3$ & $91 \pm 6.3$ & $93 \pm 11.0$ \\
\hline Non-neuropathic patients (8) & $120 \pm 15.1$ & $134 \pm 22.8$ & $113 \pm 7.4$ & $115 \pm 9.8$ & $109 \pm 8.4$ & $103 \pm 7.8$ & $95 \pm 8.8$ & $97 \pm 11.8$ \\
\hline
\end{tabular}

Values are mean \pm SEM, ${ }^{\mathrm{a}} \mathrm{p}<0.05$

Neuropathic patients are compared with non-neuropathic patients 

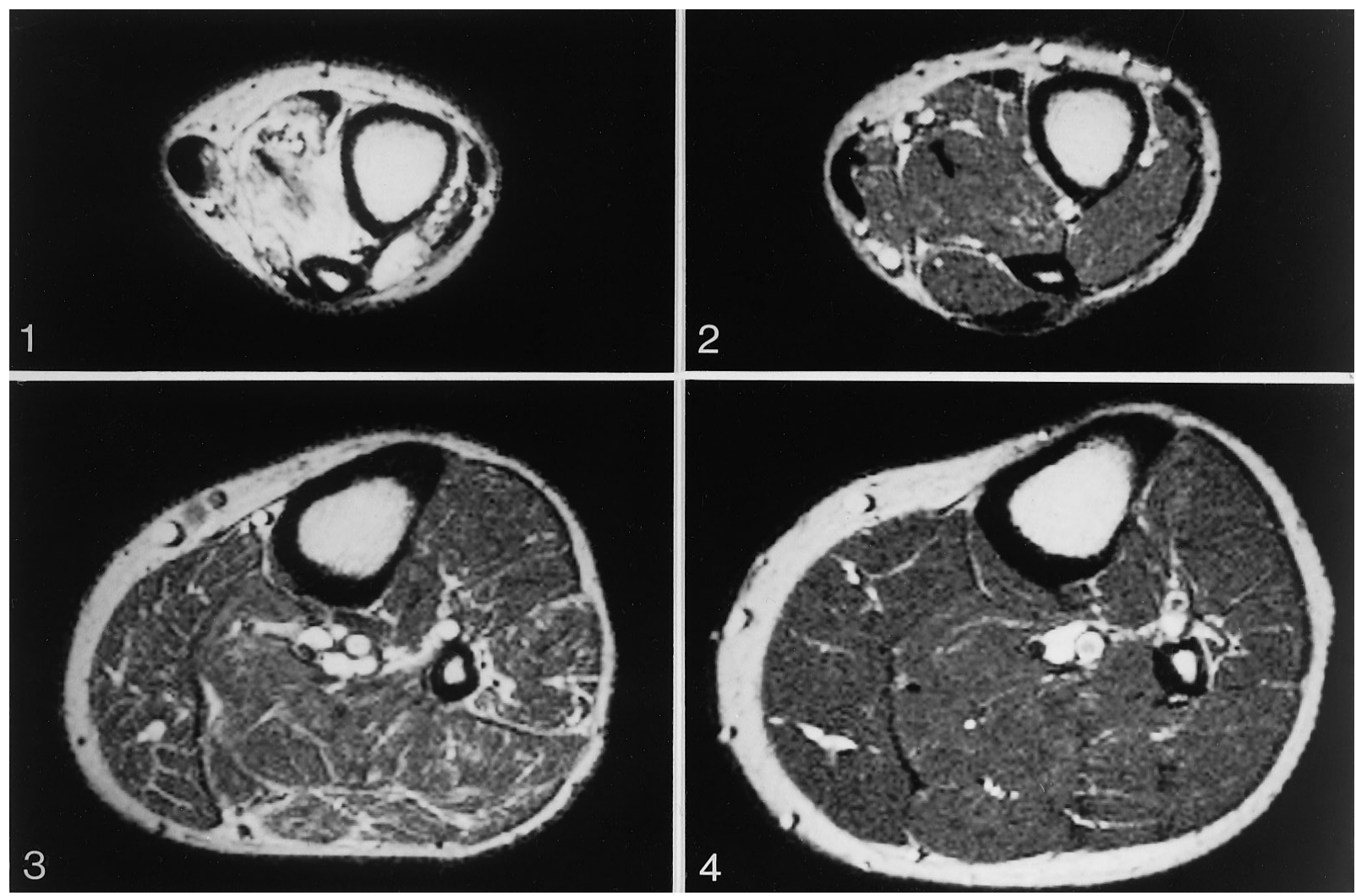

Fig. 3. Cross-sectional MR-images distally and proximally at the lower leg in a neuropathic patient (1 and 3 ) and in the matched control subject ( 2 and 4 )

$75 \pm 16.5 \mathrm{Nm} / \mathrm{dm}^{3}$ and $87 \pm 12.1 \mathrm{Nm} / \mathrm{dm}^{3}$ (mean \pm SD), respectively. The intrinsic strength of the plantar flexors was $64 \pm 13.3 \mathrm{Nm} / \mathrm{dm}^{3}$ in the diabetic group as compared to $74 \pm 9.7 \mathrm{Nm} / \mathrm{dm}^{3}$ in the control group $(p<0.05)$. The intrinsic strength of the dorsal and plantar flexors of the neuropathic patients expressed as a percentage of control subjects was $82 \pm 19.4 \%$ and $81 \pm 7.2 \%$, respectively. These values were not significantly different from the intrinsic strength of the non-neuropathic group being $92 \pm 19.4 \%$ and $93 \pm 17.4 \%$, respectively.

\section{Discussion}

In diabetic polyneuropathy sensory disturbances predominate. Recently we observed that long-term IDDM patients with symptomatic neuropathy have a considerable decrease in muscle strength at the ankle and knee [3]. The muscle weakness is related to the severity of neuropathy and is believed to be caused by neurogenic atrophy due to axonal degeneration of the motor fibres [2].

It is a classical clinical observation that diabetic patients can develop muscular atrophy. Visual inspection

and measurement of the circumference of the leg is clinically used to detect and quantitate the atrophy. In this study the external circumference was not significantly reduced at the distal part of the lower leg and only reduced approximately $10 \%$ at the mid level in the neuropathic group. In contrast the cross-sectional area of the muscle compartments was substantially reduced at both the mid and distal level of the lower leg in the neuropathic group. In addition since a part of the volume within the muscle compartments was "non-muscular tissues" the external leg circumference is clearly less sensitive for the detection of muscular atrophy than MRI. Considering the small difference in external circumference and the relatively large interpersonal variation, external measurements are insensitive for the detection and quantification of symmetrical muscular atrophy in individual patients.

For qualitative and quantitative evaluation of muscles with a non-invasive imaging technique, MRI provides the highest structural resolution without introducing any hazard to the patients [4]. In the present study the MR-images allowed the identification of muscle groups and, furthermore, at most images it was possible to isolate individual muscles within the muscle compartments. In neuromuscular diseases, MRI has primarily been used for studies of myopathies [4], but the technique has also been applied in patients with neurogenic muscle pathology [18-21]. Denervated muscles have an increased signal intensity on MR-images which enables diagnosis of even 

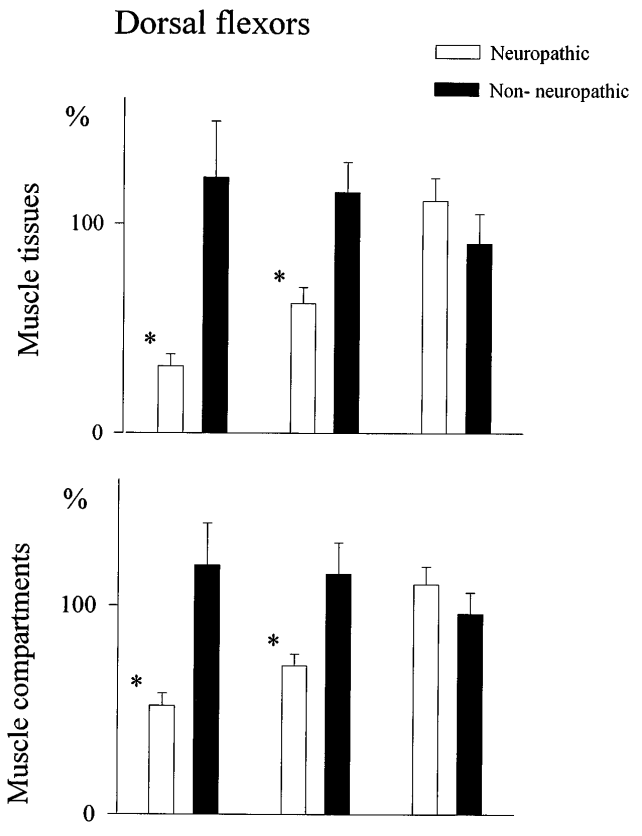

Distal crus Mid crus Proximal crus

Fig. 4. Cross-sectional area (\% of matched control subjects) of muscle tissues (above) and muscle compartments (below) of the dorsal flexors at the distal, mid, and proximal level of the lower leg (crus) in neuropathic $\square$ and non-neuropathic $\square$ patients. Data are mean \pm SEM. Values for neuropathic patients are compared with non-neuropathic patients. $* p<0.02$

isolated nerve injuries [19, 21]. Both subacutely and chronically denervated muscles have increased signal intensity while no changes can be found in acutely denervated muscles [21]. In chronically denervated muscles a close relation between the MRI increase in signal intensity and the electromyography evidence of denervation has been reported [22]. The reason for the increased signal intensity in chronic denervated muscles has not been convincingly documented. Fatty degeneration of muscle tissue and a shift in the water content from the intracellular to the extracellular compartment have been suggested [20, 23, 24]. Regardless of the mechanism, the close relation between muscle strength and volume in the present study suggests that the hyperintense areas are nonfunctional.

In diabetic patients MRI of muscles has only been used for the detection of infarction of muscles [25] whereas muscular morphology has not been studied in diabetic patients with polyneuropathy. It is evident from the findings of the present study that the impaired motor function of ankle dorsal and plantar flexors in the neuropathic patients is accompanied by atrophy and morphological changes of the muscle tissues. In addition, the neuropathic patients had a higher frequency of retinopathy and nephropathy as compared with the non-neuropathic patients indicating more severe small vessel disease (Table 1).
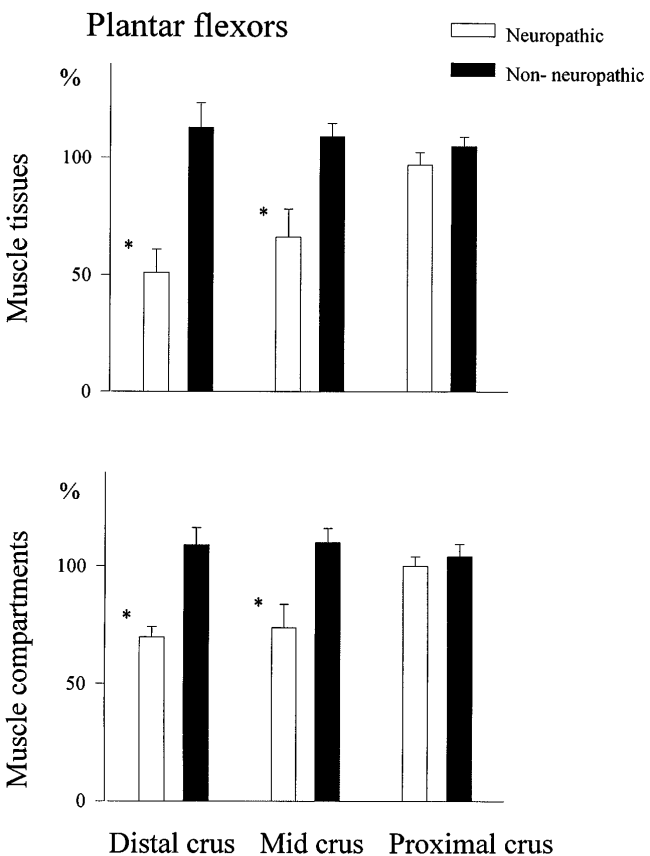

Fig. 5. Cross-sectional area (\% of matched control subjects) of muscle tissues (above) and muscle compartments (below) of the plantar flexors at the distal, mid, and proximal level of the lower leg (crus) in neuropathic $\square$ and non-neuropathic $\square$ patients. Data are mean \pm SEM. Values for neuropathic patients are compared with non-neuropathic patients. $* p<0.01$

However, none of the neuropathic patients had intermittent claudication and the dorsal foot artery was palpable in all patients. Furthermore minor differences of age between the neuropathic and non-neuropathic patients were insignificant. Consequently, the atrophy and the morphological changes of the muscles of the neuropathic patients are related to the neuropathic abnormalities whereas a direct ischaemic effect on striated muscle tissues of small vessel disease or subclinical macrovascular disease seem unlikely.

Observation of a proximo-distal gradient for the atrophy of the muscles in the lower leg is new. This topographical distribution of atrophy is comparable to the well known proximo-distal gradient of sensory disturbances in neuropathic patients [2]. The explanation of a predominant distal atrophy is given by the finding of a substantial atrophy of the flexor hallucis longus muscles situated distally in the lower leg while the gastrocnemii muscles situated proximally are unaffected. Distal muscular atrophy most likely reflects the effect of a length dependent neuropathic process within motor fibres. The finding of a more pronounced loss of volume of the dorsal flexors as compared to the plantar flexors distally at the lower leg could also be a consequence of a length dependent neuropathic process (Figs. 4 and 5). Thus, the dorsal flexors are innervated by the deep peroneal nerve which orginates from more proximal nerve 
roots (L4-S1) resulting in a slightly longer fiber pathway than for the tibial nerve innervating the plantar flexors (L5-S2).

Using an unbiased stereological method, the volume of any compartment of interest can be estimated with a coefficient of error of less than $5 \%$ pending 10 images are performed [8]. Recently it has been reported that a change in muscle volume of only $3 \%$ of the leg can be detected with MRI [26]. To our knowledge this is the first study of muscle volume in patients with neuromuscular diseases. For muscles with a variable cross-sectional area the most representative level at which to perform a scan is difficult to define. This especially applies to patients with neuromuscular disorders where pathologic processes often are unevenly distributed. Therefore, muscle volume provides a reliable measure which can be used for disease monitoring and for evaluation of the effect of treatment.

The ratio of strength to volume (intrinsic muscle strength) was reduced for the diabetic patients in comparison to control subjects. There was a tendency for a greater reduction in this ratio in the neuropathic patients although it did not reach statistical significance. A reduced intrinsic muscle strength in the diabetic patients can be caused by impaired contractility as has been found in experimentally diabetic rats [27]. Muscle contractility in diabetic patients, however, has never been studied in detail. Alternatively, a higher proportion of type 1 muscle fibers with a lower strength/volume ratio in the diabetic patients could explain the reduced intrinsic muscle strength [28, 29]. The lower intrinsic muscle strength could also be a consequence of the technique applied for muscle volume estimation. In the present study the volume was calculated by subtracting the areas of increased signal intensity within muscle compartments from total areas of muscle compartments. The cut-off level applied for increased signal intensity may have led to the inclusion of areas representing fat tissues or water. Further studies are needed to assess the contractile and histological properties of the muscles in relation to the muscle strength in diabetic patients with and without neuropathy.

Long-term, non-neuropathic IDDM patients with preserved muscle strength have normal muscle volume and normal muscle MRI signal intensity. Longterm IDDM patients with symptomatic polyneuropathy and muscle weakness have pronounced atrophy of muscles of the lower leg and abnormally increased MRI signal intensity within the muscle compartments. The atrophy progresses in a distal direction indicating that a length-dependent neuropathic process underlies weakness and loss of muscle mass.

A cknowledgements. R. Sangill, MSc, is acknowledged for excellent technical assistance. F. Schönau Jørgensen, MD, PhD and $\mathrm{K}$. Wildenhoff, MD, PhD are acknowledged for patient referral.

\section{References}

1. Dyck PJ, Kratz KM, Karnes JL et al. (1993) The prevalence by staged severity of various types of diabetic neuropathy, retinopathy, and nephropathy in a population-based cohort: the Rochester Diabetic Neuropathy Study. Neurology 43: 817-824

2. Thomas PK, Tomlinson DR (1993) Diabetic and hypoglycemic neuropathy. In: Dyck PJ, Thomas PK, Griffin JW, Low PA, Poduslo JF (eds) Peripheral neuropathy. Saunders, Philadelphia, pp. 1219-1250

3. Andersen H, Poulsen PL, Mogensen CE, Jakobsen J (1996) Isokinetic muscle strength in long-term IDDM patients in relation to diabetic complications. Diabetes 45: 440-445

4. De Visser M, Reimers CD (1994) Muscle imaging. In: Engel AG, Franzini-Armstrong C (eds) Myology. McGrawHill Inc, New York, pp. 795-806

5. Sipila S, Suominen H (1991) Ultrasound imaging of the quadriceps muscle in elderly athletes and untrained men.Muscle Nerve 14: 527-533

6. Gunreben G, Bogdahn U (1991) Real-time sonography of acute and chronic muscle denervation. Muscle Nerve 14: 654-664

7. Laroche M, Rousseau H, Mazieres B, Bonafe A, Joffre F, Arlet J (1989) Advantage of CT scan in muscular pathology. Personal cases and review of the literature. Rev Rhum Mal Osteo Articul 56: 433-439

8. Roberts N, Cruz-Orive LM, Reid NMK, Brodie DA, Bourne M, Edwards RHT (1993) Unbiased estimation of human body composition by the Cavalieri method using magnetic resonance imaging. J Microsc 171: 239-253

9. Dyck PJ, Sherman WR, Hallcher LM et al. (1980) Human diabetic endoneurial sorbitol, fructose, and myo-inositol related to sural nerve morphometry. Ann Neurol 8: 590596

10. Dyck PJ (1993) Quantitating severity of neuropathy. In: Dyck PJ, Thomas PK, Griffin JW, Low PA, Poduslo JF (eds) Peripheral neuropathy. Saunders, Philadelphia, pp. 1219-1250

11. Dyck PJ, O'Brien PC, Kosanke JL, Gillen DA, Karnes JL (1993) A 4, 2, and 1 stepping algorithm for quick and accurate estimation of cutaneous sensation threshold. Neurology 43: 1508-1512

12. Stålberg E, Falck B (1993) Clinical motor nerve conduction studies. Methods Clin Neurophysiol 4: 61-80

13. Ferner H, Staubesand J (1982) Sobotta Atlas of Human Anatomy 2. Urban \& Schwarzenberg, Munich

14. Weibel ER (1979) Stereological methods. Practical methods for biological morphometry. Academic Press, London

15. Gundersen HJ, Jensen EB (1987) The efficiency of systematic sampling in stereology and its prediction. J Microsc 147: 229-263

16. Andersen H (1996) Reliability of isokinetic measurements of ankle dorsal and plantar flexors in normal subjects and in patients with peripheral neuropathy. Arch Phys Med Rehabil 77: 265-268

17. Dyck PJ, Kratz KM, Lehman KA et al. (1991) The Rochester Diabetic Neuropathy Study: design, criteria for types of neuropathy, selection bias, and reproducibility of neuropathic tests. Neurology 41: 799-807

18. Shabas D, Gerard G, Rossi D (1987) Magnetic resonance imaging examination of denervated muscle. Comput Radiol 11: 9-13

19. Carter GT, McDonald CM, Chan TT, Margherita AJ (1995) Isolated femoral mononeuropathy to the vastus lateralis: EMG and MRI findings. Muscle Nerve 18: 341344 
20. Fleckenstein JL, Watumull D, Conner KE et al. (1993) Denervated human skeletal muscle: MR imaging evaluation. Radiology 187: 213-218

21. Polak JF, Jolesz FA, Adams DF (1988) Magnetic resonance imaging of skeletal muscle. Prolongation of T1 and T2 subsequent to denervation. Invest Radiol 23: 365-369

22. McDonald CM, Anderson MW, Nelson JO, Wineinger MA (1996) Magnetic resonance imaging versus needle electromyography for evaluation of acute and subacute denervation in skeletal muscle. Arch Phys Med Rehabil 11: 1235-1239

23. Polak JF, Jolesz FA, Adams DF (1988) NMR of skeletal muscle. Differences in relaxation parameters related to extracellular/intracellular fluid spaces. Invest Radiol 23: 107112

24. Uetani M, Hayashi K, Matsunaga N, Imamura K, Ito N (1993) Denervated skeletal muscle: MR imaging. Work in progress. Radiology 189: 511-515
25. Barohn RJ, Bazan C, Timmons JH, Tegeler C (1994) Bilateral diabetic thigh muscle infarction. J Neuroimaging 4: 43-44

26. Ferrando AA, Stuart CA, Brunder DG, Hillman GR (1995) Magnetic resonance imaging quantitation of changes in muscle volume during 7 days of strict bed rest. Aviat Space Environ Med 66: 976-981

27. Cotter M, Cameron NE, Lean DR, Robertson S (1989) Effects of long-term streptozotocin diabetes on the contractile and histochemical properties of rat muscles. Q J Exp Physiol 74: 65-74

28. Young A (1984) The relative isometric strength of type I and type II muscle fibres in the human quadriceps. Clin Physiol 4: 23-32

29. Medina Sanchez M, Rodriguez Sanchez C, Vega Alvarez JA, Menedez Pelaez A, Perez Casas A (1991) Proximal skeletal muscle alterations in streptozotocin-diabetic rats: a histochemical and morphometric analysis. Am J Anat 191: 48-56 